\title{
Double Selection Genetic Algorithm for Information Extraction
}

\author{
H.Balaji \\ JNTU Anantapur,Anantapuramu
}

\author{
A. Govardhan, Ph.D. \\ Professor and Director, School of Information \\ Technology, \\ JNTU Hyderabad
}

\begin{abstract}
Data extraction might be characterized as the undertaking of naturally concentrating occurrences of detailed classes or relations from text. This paper exhibits another preparing system focused around enhanced GA and greatest probability technique to get HIDDEN MARKOV MODEL with improved state count and its model parameters for web data extraction. This strategy defeats the deficiencies of the moderate merging rate of the HIDDEN MARKOV MODEL approach. From explores of different avenues regarding the 2100 networks removed from proposed corpus. This strategy has capacity to find ideal topology in all cases. Enhanced Genetic calculation may be utilized for web data extraction by forming a duplicate in the accompanying way as every state is connected with its group that it needs to concentrate, for example, writer or book title. Every state transmits terms from group particular dissemination. It can take in the group particular unigram conveyance and the state move probabilities from preparing information by Improved Genetic calculation mixture operations. With a specific end goal to mark another web with groups, it treats the terms from the web as perceptions and recoups the no doubt state grouping with the Viterbi calculation. In this adjusted Genetic calculation is utilized to concentrate data utilizing Hidden markov models.
\end{abstract}

\section{INTRODUCTION}

In numerous application spaces, there is the possibility to incredibly expand the utility of on-line content sources by utilizing computerized systems for mapping chose parts of the unstructured content into an organized representation. For instance, the custodians of genome databases might want to have instruments that could precisely separate data from the scientific writing about substances, for example, qualities, proteins, cells, infections, and so on. Therefore, there has been much late enthusiasm toward creating systems for the undertaking of data extraction (IE), which might be defined as naturally perceiving and concentrating occurrences of particular classes of substances and connections among elements from content sources. The internet creates accessible a significant measure of World Wide Web that's been created for man usage; this specific unfathomable amount of data seriously isn't correctly handled or perhaps examined simply by unit. Data extraction is the methodology of filling fields in a database via consequently concentrating sub-successions of intelligible web. Illustrations incorporate concentrating the area of a gathering from an email message, or concentrating the name of the procured organization in a newswire article around an organization takeover [1].

\section{LITERATURE SURVEY}

Most precedent effort in the ground of data recuperation using Hidden markov models included hand gathered Hidden markov models, for instance, those portrayed in. It associated Hidden markov models with machine learned parameters to the errand of finding names and other non recursive substances in substance. The venture which completed their hand-coded HIDDEN MARKOV MODEL, Nymble, fulfilled a high F-score of 90-95.
The Hidden markov models executed by Leek is furthermore complicatedly delineated, for the task of concentrating (quality name, chromosome region) sets from trial papers in the restorative space. For sure, Hidden markov models have been joined viably in various fields related in nature to information extraction. In pharmaceutical, Hidden markov models are a noteworthy gadget for concentrating basic DNA segments from genome data bases in. Markov used to replica mapping between trajectory parcels in acoustic space to phonetic syllables.

Accomplishment of Hidden markov models depend on upon the way that their pictorial illustration empowers human directed model layout, however the vicinity of EM parameter estimation figuring's license data ward learning. Significantly more relevant to this wander is the issue of information recuperation using Hidden markov models whose structure is controlled by some machine learning computation. The crucial establishment of this endeavor is found in the work of authors in HIDDEN MARKOV MODEL adjusting through stochastic upgrade. Few authors showed a by and large diverse philosophy to HIDDEN MARKOV MODEL structure learning: Start from the most ensnared structure and use consolidating techniques to explore the structure space. Such state uniting technique is moreover used by authors.

\section{PROPOSED WORK}

A direct Genetic Algorithm cycle contains four operations Fitness assessment, choice, hereditary operations, and substitution. In a fundamental Genetic Algorithm cycle, there exists a masses pool of chromosomes. Chromosomes are encoded sort of the potential results and all Genetic Algorithm operations beside well being evaluation to be performed with this appearance of results. At the outset, the masses are made aimlessly and the well being estimations of each and every one of chromosomes are surveyed by determining the objective limit in the decoded kind of chromosomes. After the in articulation of the people pool, the Genetic Algorithm headway cycle is begun. The mating pool is formed by selecting a couple of chromosomes from people. This pool of chromosomes is used as the people for the innate operations to make the descendants or the sub populace. The health estimations of the family are in like manner surveyed. Toward the end of the period, a couple of chromosomes in the people will be supplanted by the family according to the substitution plan [10] [12]. The above time is repeated until the end premise is met. By duplicating the basic decision and genetic operations, this philosophy will positively leave best chromosomes or the outstandingly streamlined responses for the issue in the last populace.

\subsection{Encoding}

The chromosome is normally communicated in a series of components and every component of which is known as a quality. As per the issue determinations, a quality could be characterized as the kind of double, genuine number, or different structures. Bit string encoding is the most excellent methodology 
utilized by GA scientists because of its effortlessness and traceability.

\subsection{Fitness Function}

This is used to focus certainty level of the upgraded answers for issue. Ordinarily, there is a wellness worth connected with every chromosome. A higher wellness worth implies that chromosome or result is more upgraded to a subject while a lower estimation wellness shows fewer advanced chromosome. Wellness qualities are aftereffects of target capacity. As probability $\mathrm{P}[\mathrm{o} \mid \lambda]$ is a fitting paradigm utilized within the destination capacity to focus the nature of the chromosomes. The likelihood $\mathrm{P}[\mathrm{o} \mid \lambda]$ is computed by the greatest probability technique.

\subsection{Improved Selection}

a) Pre selection

b) Post selection

The determination instrument to decide the guardian chromosomes from populace and structures the mating pool. Determination instrument imitates the survival of the fittest component in nature. It is normal that a fitter chromosome gets a higher number of posterity and in this manner has a higher shot of getting by in the consequent development while the weaker chromosomes will pass on end. A virtual wheel is utilized within this choice system. Every chromosome in the populace is connected with a part in the virtual wheel. As indicated by the wellness estimation of the chromosome, the part will have a bigger region when the comparing chromosome has a finer wellness worthwhile a lower wellness quality will prompt a more modest division.

$\mathrm{p}_{\mathrm{i}=} \mathrm{F}_{\mathrm{i}} / \sum_{i=1}^{M} F_{i}, i=1,2, \ldots \ldots, M$

Where $\mathrm{Pi}$ is the normalized fitness value of a Mth chromosome selected form population and $\mathrm{Fi}$ is fitness value of a chromosome in the population. In the pre selection list of chromosomes are selected. In post selection rank based selection uses a fitness value of the chromosomes to sort chromosomes from highest to lowest.

\subsection{Crossover}

It is used to join subparts of the parents to deliver posterity. This contains a few parts of guardian hereditary materials. They chose parents in all probability the fitter chromosomes. It might be seen that this administrator is intended to consolidate the streamlined hereditary materials in the parents jointly to deliver extra gained posterity.

\subsection{Mutation}

It gives worldwide seeking ability to GA by arbitrarily adjusting the estimations of qualities in the chromosomes. Prior to the alteration of a model parameter, transformation rate will be contrasted with an arbitrarily produced likelihood with test if the change rate is bigger than or equivalent to the haphazardly created likelihood.

\subsection{Information Extraction}

Genetic algorithm using hidden markov model may be utilized for web data mining by forming a representation in accompanying way in which each one state that is connected with a group that it needs to concentrate, for example, title, writer or book title. Each one state emanates terms from a group particular unigram conveyance. It can obtain in group particular unigram conveyance and state move probabilities from preparing information by Genetic algorithm Hidden Markov Model crossover operations. Keeping in mind the end goal to name another web with classes, It treats terms from the web as perceptions and recoup the in all likelihood state arrangement with Viterbi calculation. Express that delivers each one saying is group tag for that statement.

\section{RESULTS}

Experiments are conducted by taking different datasets. Experimental results show significant improvement. Proposed system gives better results in terms of precision and recall.

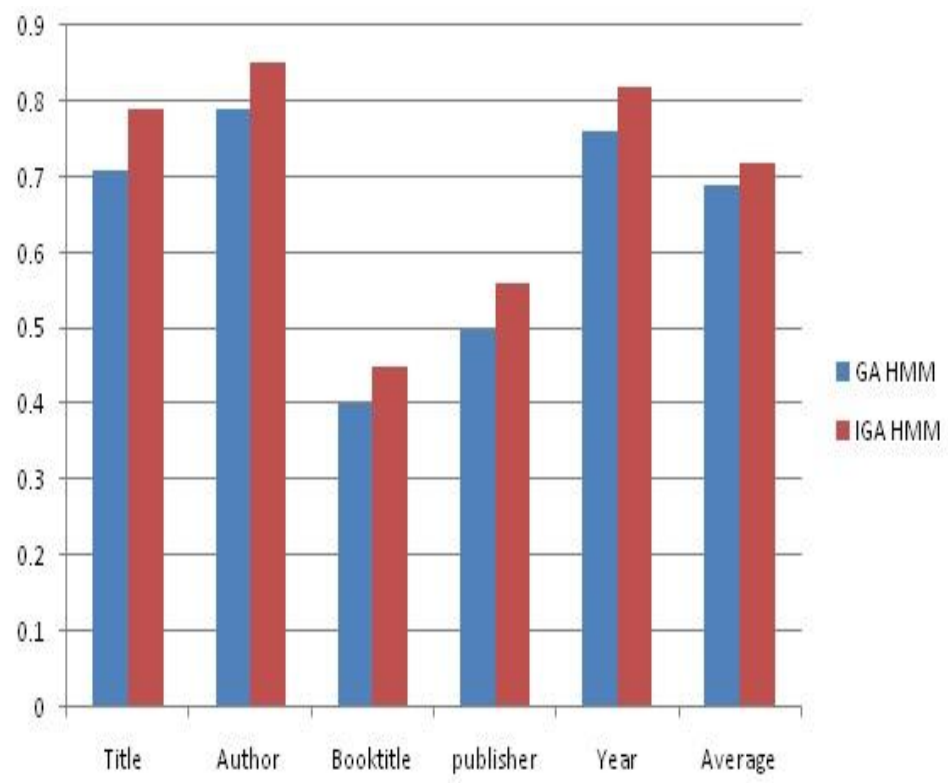

Fig1.Recall

As shown in Fig1 double selection algorithm improves ignificant change in recall when compared to existing method.

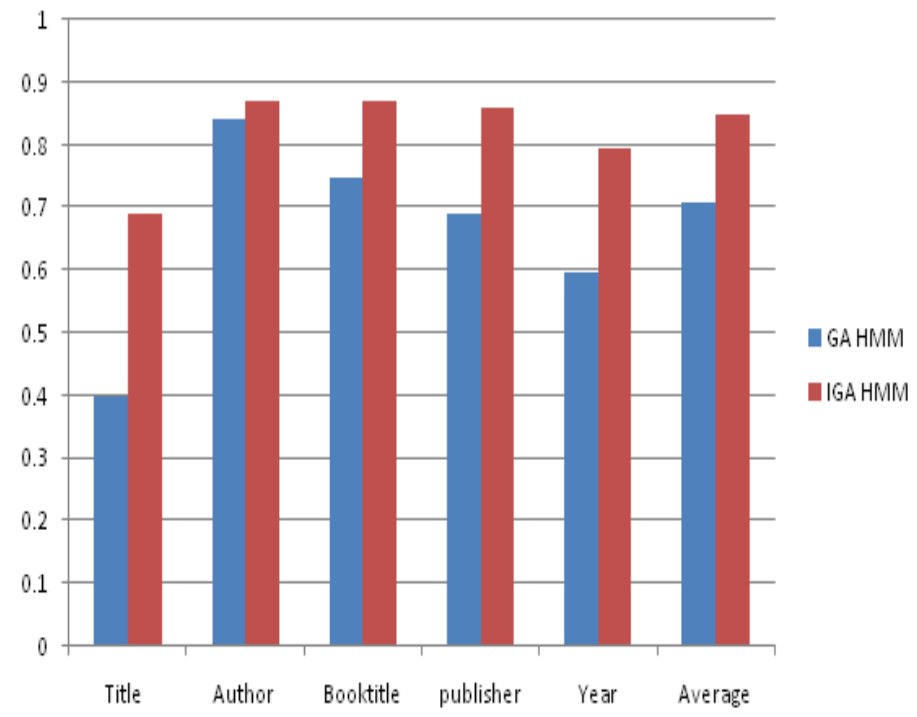

Fig2. Precision

As shown in Fig2 double selection algorithm improves significant change in precision when compared to existing method.

\section{CONCLUSION}

The proposed procedure is another Hidden markov model focused around enhanced hereditary calculations for web data mining. This strategy discovers great HIDDEN MARKOV 
MODEL topology and additionally its representation parameters. From tries different things with the 2100 networks extricated from corpus, proposed plan has the capacity discover the best states in all cases. From the exploratory results, enhanced hereditary administrator can find the ideal state count even on an account of an uneven early dispersion of the quantity of events of the chromosomes with distinctive state count. This showed that proposed administrator is a solid methodology for discovering the ideal state count in HIDDEN MARKOV MODEL. By utilizing an extremely straightforward hereditary administrator, the enhanced GA can find the ideal state count in the expression demonstrate effectively. The experimental results also state that proposed technique is giving better results compare to existing methods.

\section{REFERENCES}

[1] D. Freitag and A. Mccallum, Information extraction with HIDDEN MARKOV MODELs and shrinkage. Proceedings of the AAAI'99 Workshop on Machine Learning for Information Extraction, pp.31-36, 1999.

[2] D. Freitag and A. Mccallum, Information extraction with HIDDEN MARKOV MODEL structures learned by stochastic optimization. Proceedings of the Eighteenth Conference on Artificial Intelligence, pp.584-589, 2000.

[3] K. Seymore , A. Mccallum and R. Rosenfeld, Learning hidden markov model structure for information extraction. AAAI'99 Workshop on Machine Learning for Information Extraction, pp.37-42, 1999.

[4] D. Freitag, A. Mccallum and F. Pereira, Maximum entropy markov models for information extraction and segmentation. Proceedings of ICML-2000, pp.591-598, 2000 .
[5] D. Bouchaffra and J. Tan, Structural hidden markov models using a relation of equivalence:

[6] R.J. Mooney and U.Y. Nahm, Text mining with information extraction. Multilingualism and Electronic language Management, Proceedings of the 4th International MIDP Colloquium, pp.141-160, 2005.

[7] X.H. Phan, S. Horiguchi and T.B. Ho, Automated data extraction from the web with conditional models. Int.J. Business Intelligence and Data mining, 2:191-209, 2005.

[8] S. Kwong, C.W. Chan, K.F. Man and K.S. Tang, Optimization of HIDDEN MARKOV MODEL topology and its model parameters by genetic algorithms, Pattern Recognition,34:509-522, 2001.

[9] Q.Y. Hong and S. Kwong, A genetic classification method for speaker recognition. Engineering Applications of Artificial intelligence, 18:13-19, 2005.

[10] A. Asllani and A. Lari, Using genetic algorithm for dynamic and multiple criteria web-site optimizations. European Journal of Operational Research, 176:1767-1777, 2007.

[11] M. Caramia, G. Felici and A. Pezzoli, Improving search results with data mining in a thematic search engine. Computers \& operations Research, 31:2387-2404, 2004.

[12] H. Zhon, Y.C. Feng and L.M. Han, The hybrid heuristic genetic algorithm for job shop scheduling. Computers \& Industrial Engineering, 40:191-200, 2001.

[13] Jiyi Xiao Lamei Zou Chuanqi Li, "Optimization of Hidden Markov Model by a Genetic Algorithm for Web Information Extraction". 